\title{
Enhanced frequency analysis on a vibrated tumor with a compression cylinder
}

\author{
Satoshi Miura ${ }^{1 *} \mathbb{B}$, Hidekazu Ishiuchi ${ }^{1}$, Yuta Shintaku' ${ }^{1}$, Victor Parque ${ }^{1}$, Ayako Torisaka ${ }^{2}$ and Tomoyuki Miyashita ${ }^{1}$
}

\begin{abstract}
Breast cancer diagnosis has been mostly accomplished through imaging. These methods have great advantages in being able to detect the presence and location of breast cancer. However, it is difficult to distinguish between a benign and malignant tumor located in a deep position because both tumor types look similar. In this paper, tissue including the tumor from skin was vibrated using a compression cylinder, to analyze the frequency difference for distinguishing tissue type. Before distinguishing between a benign and malignant tumor, it is necessary to validate that the difference between normal tissue and tumor can be distinguished. The objective of the study is to validate the feasibility to emphasize the frequency differences in a $10.0 \mathrm{~mm}$ or greater deep tumors during vibration by pushing a cylinder towards the deep tumor. A phantom model and finite element analysis model were constructed to simulate the breast. In the experiment, air was injected into the phantom and the displacement was measured. The frequency response for distinction of tissue types was analyzed and it was found that the displacement difference rate was over $50 \%$ at a frequency of $130 \mathrm{~Hz}$ when the cylinder was pushed into the sample as opposed to when not pushed in. Changes in displacement were measured according to the distance between the tumor and vibration point using finite element analysis. When the measurement and vibration points were on the center of the tumor, the difference in the resonance point was at its largest $(5.5 \mathrm{~Hz})$. Results show that the position of a tumor could be easily and rapidly detected by vibrations from a cylinder pushed into the diagnostic site.
\end{abstract}

Keywords: Vibration analysis, Breast cancer diagnosis, Resonance frequency analysis

\section{Background}

The occurrence of cancer is increasing every year and it continues to be a leading cause of death [1]. More than $1,300,000$ women are diagnosed with breast cancer worldwide each year. There are many tumor types [2] such as Ductal carcinoma in situ [3], Invasive micropapillary carcinoma [4], Invasive lobular carcinoma [5] and mucinous Carcinoma [6], apart from the degree of progression. The simplest tumor discrimination are two type as following [5]. One is a benign tumor that does not transfer to other organs, so it is typically not necessary to operate. The other is a malignant tumor that transfers to and destroys other organs. A malignant organ attacks other normal organs by infiltrating it, so early operation

\footnotetext{
*Correspondence: miura.s@aoni.waseda.jp

1 Department of Modern Mechanical Engineering, Waseda University,

3-4-1 Okubo, Shinjuku, Tokyo 169-8555, Japan

Full list of author information is available at the end of the article
}

is necessary. Therefore, the early diagnosis of cancer remains vitally important for a good prognosis and positive treatment outcomes. However, tumor classification method is not established because it's difficult to distinguish between benign and malignant [7].

\section{Related works}

Screening technologies have been developed in a variety of methods. Tumor detection usually involves imaging diagnosis methods such as scatter X-ray imaging [8], mammography [9], ultrasound [10], and magnetic resonance imaging (MRI) [11]. In an experimental approach, acoustic signals have also been used to localize the position of tumors [12]. Other studies have investigated the viscoelastic characterization of normal and tumor tissue [13]. These technologies have great advantages of being able to detect the presence and location of cancers. However, it is difficult to distinguish between a benign and malignant tumor [14-16], as they can look similar. 
Therefore, it is challenging for a surgeon to distinguish cancer types through the use of imaging technologies alone.

Viscoelasticity is the difference between a benign and malignant tumor. When the tissue greatly deforms, a malignant tumor becomes harder than a benign one. Using these differences, Wang et al. [14] studied the difference deformation in tumor deformation when compressing the skin to distinguish tumor types using elastography and mammography [15]. Umut proposed a quantification method for percussion [16], vibrating the livers of animals with an impulse hammer to compare tissue properties according to their frequency responses. Kashif [17] analyzed the vibrated breast images to detect changes in stiffness. They reported that with respect to frequency, elastic moduli (storage elastic modulus, loss modulus) had different mechanical properties between normal and diseased tissues. Yamakawa and Shiina [18] also evaluated tissue viscoelasticity using vibration. The vibration method is similar to the ultrasonography in terms of the principle. Although ultrasonography used around $3 \mathrm{MHz}$, the vibration is $1.7 \mathrm{~Hz}$. The lower frequency vibration is easier to happen the resonance phenomena with the breast in the tissues scale, not particle scale. However, there are few studies that distinguish a $10.0 \mathrm{~mm}$ or greater deep tumor in tissue from surface because it is too deep for deformation and vibration on the tissue surface to show differences in tumor types. Generally, although most researches discuss about the surface position of the breast cancer [19], the breast cancer depth is important because the breast cancer often come around the Mammary gland [20, 21]. Since mammary grand is deeply located in the breast, it's important to distinguish the type of the breast cancer located deeply.

A study on needle insertion revealed a similar problem with the tumor moving in the tissue. To capture the movement of the deep tumor, Kobayashi et al. [22] made it difficult to move tumors by inserting another probe. They inserted the needle from the skin after pushing with another probe to make it difficult for the tumor to move within the tissue. Because the tumor may become harder based on loading, they anchored the tumor to make it easier to insert by push another probe in advance. They called the approach the "Preloading Method" and obtained an accurate insertion. Although this method of pushing another probe has been used in the field of needle insertion, it has not been used to distinguish tumor types.

\section{Objective}

This paper presents a novel method to emphasize the frequency differences in a $10.0 \mathrm{~mm}$ or greater deep tumors during vibration by pushing a cylinder towards the deep tumor, as shown in Fig. 1. The hollow cylinder has two advantages like as Fig. 1. One is shortening the distance to the tumor from skin. The other is that the tumor moves difficulty since the tissue is harder when pushed. Therefore, the difference in frequency increases enough to show the skin. The proposed technology is expected to use a simple discrimination method between benign or malignant after the tumor size and depth were detected by other method such as ultrasound, X-ray, and MRI. On the assumption that the tumor size and depth were known, we analyze the tumor type using vibration.

Before distinguishing between benign and malignant tumors, it is necessary to validate that we can distinguish between normal tissue and tumors using the proposed method. In this paper, we validate the feasibility of vibration analysis with a compression cylinder method, to distinguish between normal site and tumors by measuring the differences in resonance points. In the experiment, we measure the displacement of two breast phantoms, with and without a simulated tumor vibrating with an air-injector. This can distinguish normal tissue from the simulated tumor using frequency analysis. We compare displacement changes with and without a compression cylinder to analyze the differences in frequencies at peak compliance. Using finite element analysis, we construct a simulated breast model to study displacement changes as a position relationship between the tumor and the loading point. Finally, we investigate the idea that the closer the load point is to the tumor, the greater displacement difference will be.

\section{Materials}

\section{Breast model}

For simulating the viscoelastic test of human breast, the phantom is generally used in medical fields [23-26]. Thus, we constructed test pieces made out of phantom material to simulate the viscoelasticity of breast tissue

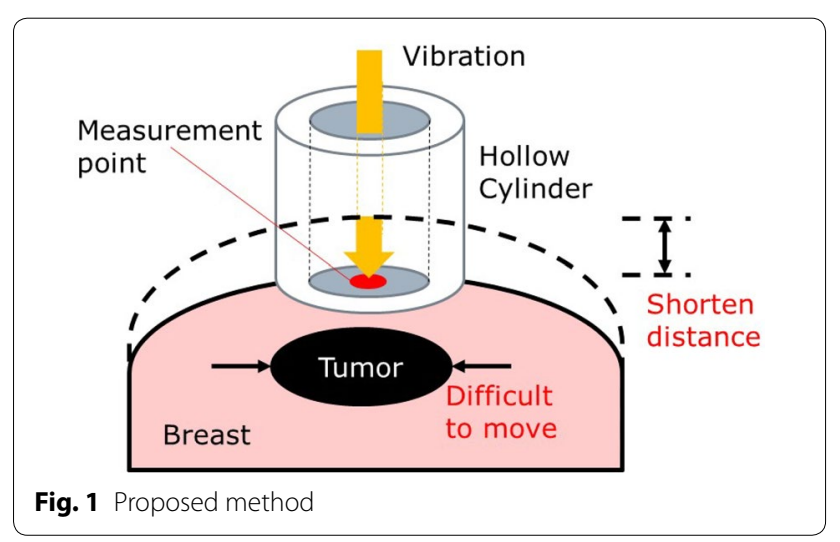


and cancer. Although there are some standard phantom models including tumor by some phantom manufacturers, it's difficult to analyze the model using finite element analysis. To validate the feasibility of the vibration response analysis method as a pilot study, the cubic shape phantom was made because the simple shape is easier to analyzed than complex breast shape.

Some reported that the elastic modulus of breast tumor is three times higher than that of normal tissue $[27,28]$. The elastic modulus of normal tissue was $7.5-14.3[\mathrm{kPa}$, that of the tumor was $11.3-46.0[\mathrm{kPa}]$. Based on these values, we adjusted the composition ratio of the silicone rubber so that the longitudinal elastic modulus of the tumor was three times that of the normal tissue. We used this experiment to validate the nonlinearity of the phantom containing a simulated tumor.

We made the phantom model using silicone rubber (KE-1603A, KE-1603B, ShinEtsu Co., Gunma, Japan) and silicone oil (KF-96-50cs, ShinEtsu). We adjusted the composition ratio of the silicone rubber according to:

$$
\text { Mixing ratio of silicone oil }[\%]=\frac{M_{0}[g]}{M_{A}[g]+M_{B}[g]}
$$

where $M_{o}[g], M_{A}[g]$, and $M_{B}[g]$ are the masses of silicone oil and silicone rubbers KE-1603A and KE-1603B, respectively. We constructed the test piece using an acrylic mold. The test piece had a cylindrical shape with a thickness of $5 \mathrm{~mm}$ and a diameter of $20 \mathrm{~mm}$.

The normal tissue for the phantom was made using silicone rubber with a mixing ratio of silicon oil $240 \%$, while the simulated tumor tissue was made with silicone rubber with a mixing ratio of silicon oil $150 \%$. For the shape, we constructed the tumor as a sphere and the normal tissue as a rectangular parallelepiped by an acrylic model. To prepare the phantom with a simulated "tumor," a portion containing no "tumor" was constructed up to a height of $20 \mathrm{~mm}$. After curing the portion, spheres of the prepared simulated tumor tissues and the remaining normal tissue were added to the mold. Phantoms with and without simulated tumors are shown in Fig. 2. The distance

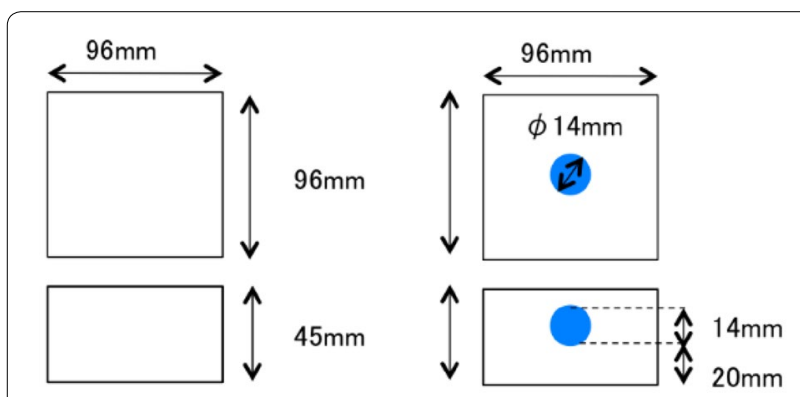

Fig. 2 Dimensions of the phantoms without (left) and with a tumor from the surface to tumor is $11 \mathrm{~mm}$. A simulated tumor was colored with the colorant KE-COLOR-BR, with the proportion of colorant set at $1 \%$ so as not to affect the physical properties of the material. The tumor diameter was $14.0 \mathrm{~mm}$ because the tumor size less than $20.0 \mathrm{~mm}$ is early breast cancer [19].

\section{Finite element analysis}

We constructed a simulated breast model to analyze the frequency response for distinction of the simulated tissue types such as normal tissue or tumor. We performed analyses on two issues; the first was the validation of the difference in mechanical compliance by pushing the cylinder into the phantom. The other was to investigate changes in displacement and compliance according to the distance between the tumor and vibration point.

The model used for the analysis was the same size as the phantom. The analysis conditions are shown in Table 1. We fixed the model with 6 degrees of freedom at the bottom. Figure 3 shows the finite element model.

\section{Table 1 Analysis conditions of the phantom}

\begin{tabular}{|c|c|}
\hline Fixation degree condition DOF & 6 \\
\hline Air injection load kPa & 1.27 \\
\hline Air injection point mm & $\begin{array}{l}\text { Center portion } \\
\text { with a diam- } \\
\text { eter of } 10\end{array}$ \\
\hline Depth of the pushing cylinder $\mathrm{mm}$ & 5.0 \\
\hline Element count & 21,942 \\
\hline Number of contacts & 34,085 \\
\hline Coefficient of mass matrix in Rayleigh attenuation a & 10 \\
\hline Coefficient of stiffness matrix in Rayleigh attenuation $\beta$ & $1.762 \times 10^{-4}$ \\
\hline Mode damping ratio $\zeta_{1}, \zeta_{2}$ & $0.438,0.448$ \\
\hline
\end{tabular}



Fig. 3 Finite element model. The depressed area was set because the hollow cylinder was pushed in the experiment 
When performing the harmonic response analysis while pushing the cylinder, we defined the preload in static analysis before the dynamic analysis. We developed the mesh in Patran software (MSC Software Co., Tokyo, Japan). A phantom containing a tumor was created as a contact body by sharing nodes. We calculated the coefficient of the Rayleigh attenuation and the mode-damping ratio from the experimental results.

\section{Methods}

\section{Air-compressor experiment}

Figure 4 shows the experimental setup. In the experiment, we used air injection to vibrate the phantom, because the air injection is safer than hammering for vibrating the human body. We measured the displacement of the phantom during vibration to test for differences in compliance between an only normal tissue and a including simulated tumor. Air injection via an SR-045 compressor (Fujiwara Inc., Japan) was used to vibrate the phantom. The air compressor was controlled by an microcomputer and a solenoid valve allowing injection of air for just $10 \mathrm{~ms}$. The injection port diameter was $5 \mathrm{~mm}$. The injection position was $95 \mathrm{~mm}$ above the phantom. The air injection load was $1.27 \mathrm{kPa}$. We measured the displacement using the sensor head (LK-G405, KEYENCE, Osaka, Japan) and controller unit (LK-G3000, KEYENCE, Osaka, Japan). The range of LK-G405 is 70-100 $\mathrm{mm}$ at sampling time $20 \mu \mathrm{s}$. The space resolution is $0.01 \mu \mathrm{m}$. The cylinder was fixed by the fixation device and used to push the phantom. The cylinder's specification is shown in Table 2.

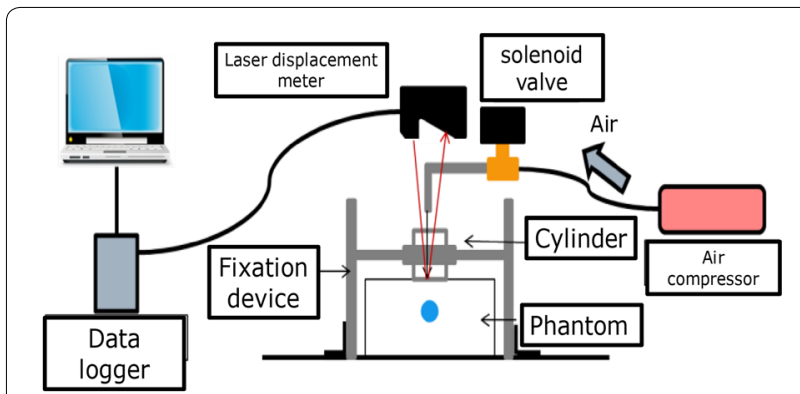

Fig. 4 Experimental setup

Table 2 Cylinder parameters

\begin{tabular}{ll}
\hline Specification & Value \\
\hline Material & Aluminum \\
Outside diameter mm & 32 \\
Thickness mm & 1.5 \\
Height mm & 500 \\
\hline
\end{tabular}

We had the solenoid valve inject air for $10 \mathrm{~ms}$ while the laser displacement sensor measured the displacement of the phantom surface. The time-displacement data were Fourier-transformed, and we then divided the displacement component by the force component to calculate the frequency response. We performed the experiment under the following four conditions:

(1) Not including the simulated tumor, no cylinder.

(2) Including the simulated tumor, no cylinder.

(3) Not including the simulated tumor, with cylinder.

(4) Including the simulated tumor, with cylinder.

We compared the compliance under these conditions, repeating the experiment 3 times for each condition. We calculated the averaged value of the results of 3 times trials in each graph. When the cylinder was used, we pushed it $5 \mathrm{~mm}$ into the phantom.

\section{Finite element analysis experiment}

Using finite element analysis, we carried out two experiments. One validated the vibration analysis with the pushing cylinder method using a simulation. We compare the resonance frequency with and without a pushed cylinder. The air injection load and the pushing cylinder depth into the model were the same as the real experiment. The second experiment analyzes the relationship between the load point and tumor position. We analyzed the displacement changes in respect to the load point changes. We analyzed the frequency peak to study the change of the frequency according to the relative load point position from the tumor shown in Fig. 5 and Table 3.

\section{Results}

Air compressor experimental results

Figure 6 shows the response frequency with no cylinder; results are the average of three trials. Figure 7 shows the response frequency when the cylinder was used. From

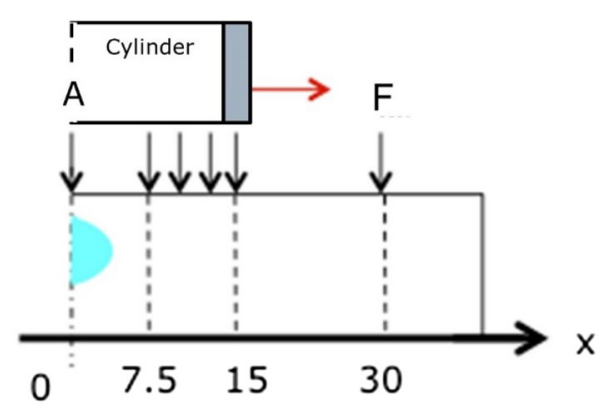

Fig. 5 Changes to the vibration point. The zero is center of the simulated tumor. $X$ value shows the distance between the measurement point and the center of the tumor 
Table 3 Vibration points

\begin{tabular}{lll}
\hline Point & $\begin{array}{l}\text { Distance } \\
\text { from the center } \\
\text { of the tumor } \mathbf{~ m m}\end{array}$ & Characteristic \\
\hline A & 0.0 & Center of the tumor \\
B & 7.5 & Inside the tumor (half the tumor) \\
C & 11.5 & Inside the tumor (outside the tumor) \\
D & 13.5 & Inside the tumor (far) \\
E & 15.0 & Under the cylinder \\
F & 30.0 & Outside the cylinder \\
\hline
\end{tabular}

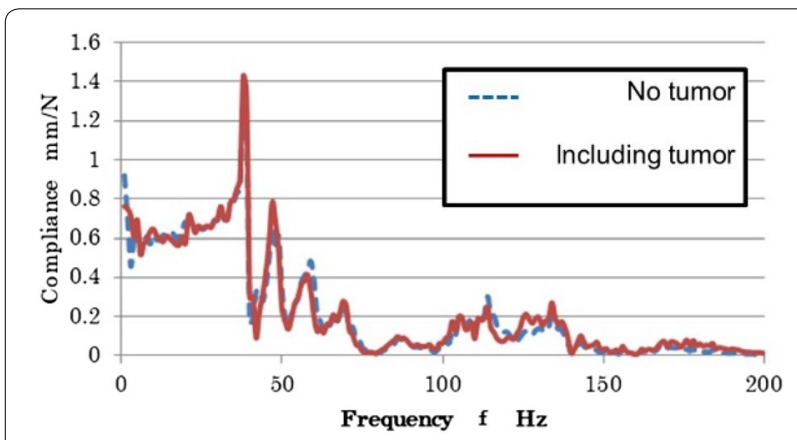

Fig. 6 Frequency response with no cylinder

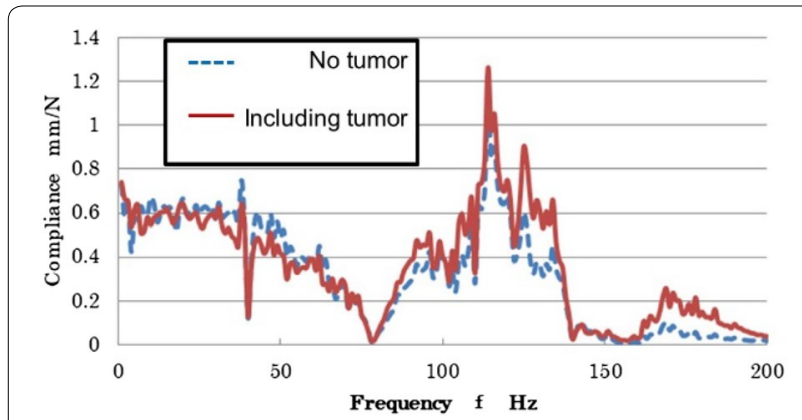

Fig. 7 Frequency response using cylinder

Figs. 6 and 7, we calculated the displacement difference rate according to:

$$
D=\frac{x_{\text {including }}-x_{\text {none }}}{x_{\text {none }}} \times 100 \%,
$$

where $D$ is the displacement difference rate, $x_{\text {including }}$ is the displacement including the simulated tumor, and $x_{\text {none }}$ is the displacement without the simulated tumor. Figures 8 and 9 show the displacement difference rates without and with the use of the cylinder, respectively. Figures 8 and 9 shows the enough frequency ranges to see the change. In Fig. 8, the displacement difference rate is less than $30 \%$, while in Fig. 9 the displacement difference rate is over $50 \%$ at $130 \mathrm{~Hz}$ with the use of the cylinder.

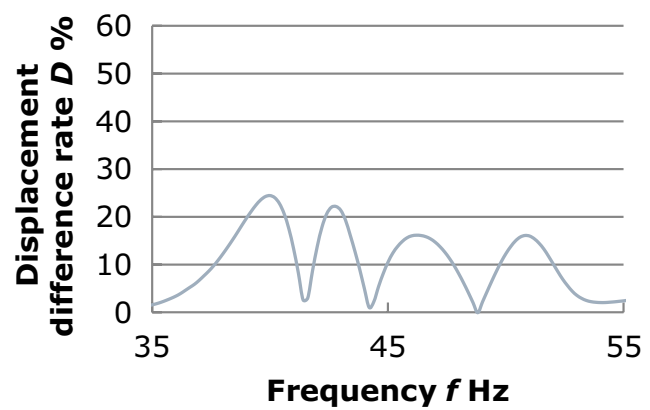

Fig. 8 Displacement difference with no cylinder

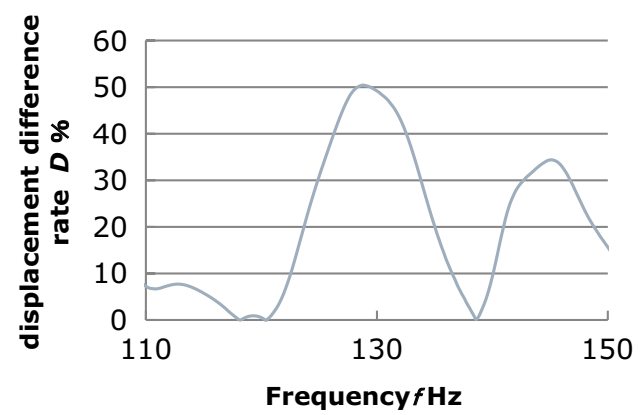

Fig. 9 Displacement difference using cylinder

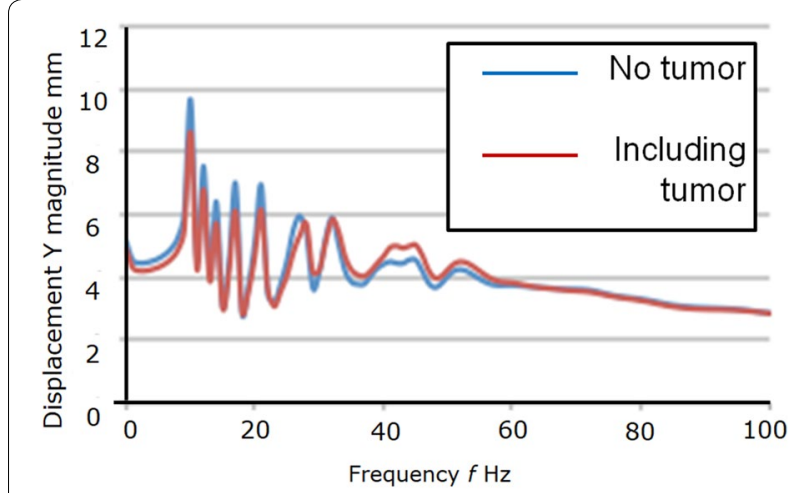

Fig. 10 Displacement without cylinder

Finite element analysis results Validation of the vibration analysis with a pushing cylinder method

We analyzed the displacement with no cylinder and when the cylinder was pushed into the simulated breast model, as shown in Figs. 10 and 11. From these data, we calculated the displacement difference rates shown in Fig. 12. Without cylinder, the displacement difference rate was $<15 \%$, while with the cylinder the displacement difference rate was $>45 \%$ at a frequency of $60 \mathrm{~Hz}$. 


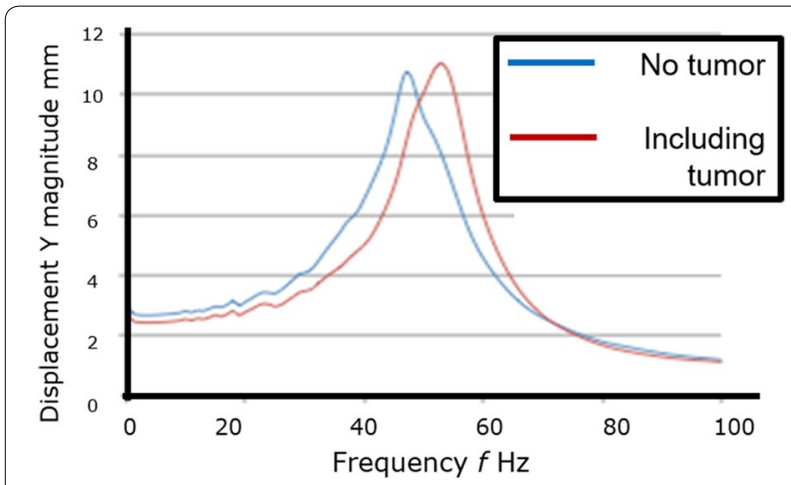

Fig. 11 Displacement by pushing the cylinder

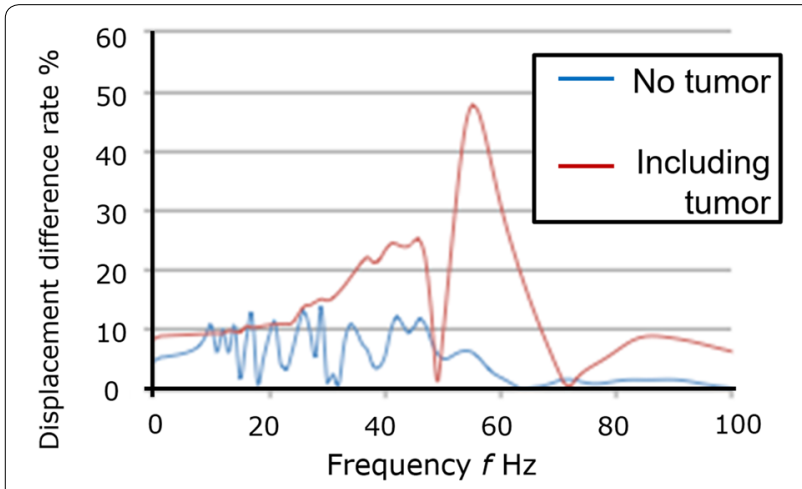

Fig. 12 Displacement difference rate with a pushing cylinder

\section{Position relationship between load point and tumor}

The results of the analysis are shown in Table 4 and Fig. 13. The peak frequency changed according to the distance between the load point and measurement point. Without the tumor, peak frequency was always approximately $47-48 \mathrm{~Hz}$, with an average of $47.5 \mathrm{~Hz}$. At the center of the tumor, the differences in the peak frequencies with and without the tumor were $5.5 \mathrm{~Hz}$. At the inside of the tumor, the difference in the peak frequency was $2.5 \mathrm{~Hz}$, while at the other points the difference was $\pm 0.5 \mathrm{~Hz}$.

\section{Discussion}

In Figs. 6 and 7, we see that the resonance frequency is different. The resonance frequency with a cylinder was larger enough to conform the tissue difference than without a cylinder. This indicates that the pushing cylinder causes the difference in resonance frequency. In addition, from Figs. 8 and 9, the $D$ is larger with a pushing cylinder than without cylinder. This suggests that pushing a cylinder would amplify the displacement difference rate to more easily distinguish the normal tissue or tumor.

Furthermore, we can also evaluate the effectiveness of the vibration analysis with a pushing cylinder method. From Figs. 10, 11 and 12, the waveform of the displacement to frequency varies greatly. In Fig. 12, $D$ differs more than $30 \%$ between tumor and no tumor using a pushing cylinder. The results elucidate the effect of pushing a cylinder on finite element analysis.

Finally, we analyzed the influence of the relative position between the load point and tumor. Figure 13 shows the displacement at each measurement point. The results indicate that the closer the load point is to the tumor, the greater the displacement difference in the peak frequency is between samples with and without a tumor. This suggests that the measurement and load points with the greatest difference in peak frequency are located on the tumor. Additionally, when the measurement and load points were removed from the center of the tumor, the difference in peak frequencies was within $1 \mathrm{~Hz}$. This suggests that we could predict tumor size from changes in peak frequency difference.

From these results, we confirmed that a pushing cylinder makes a difference in the resonance frequency of vibration analysis to diagnose a $10.0 \mathrm{~mm}$ or greater deep tumor presence and position. The hollow cylinder would have two advantages. One would be shortening the distance to the tumor from skin. Resonance frequency is larger when the excitation point is closer to the tumor. The other would be that the tumor moves difficulty since the tissue is harder when pushed. Resonance frequency is also larger when the tissue is harder because the vibration is easy to transfer. Therefore, the

Table 4 Frequencies with and without a tumor

\begin{tabular}{lll}
\hline Measurement point & Peak frequency Hz & Difference from average \\
of no tumor (47.5 Hz) Hz & No tumor & Tumor \\
\hline (A) Center of the tumor & 47 & 53 \\
(B) Inside the tumor (Half the tumor) & 48 & 50 \\
(C) Inside the tumor (Outside the tumor) & 47 & 48 \\
(D) Inside the tumor (Far) & 48 & 48 \\
(E) Under the cylinder & 48 & 48 \\
(F) Outside the cylinder & 47 & 47 \\
\hline
\end{tabular}




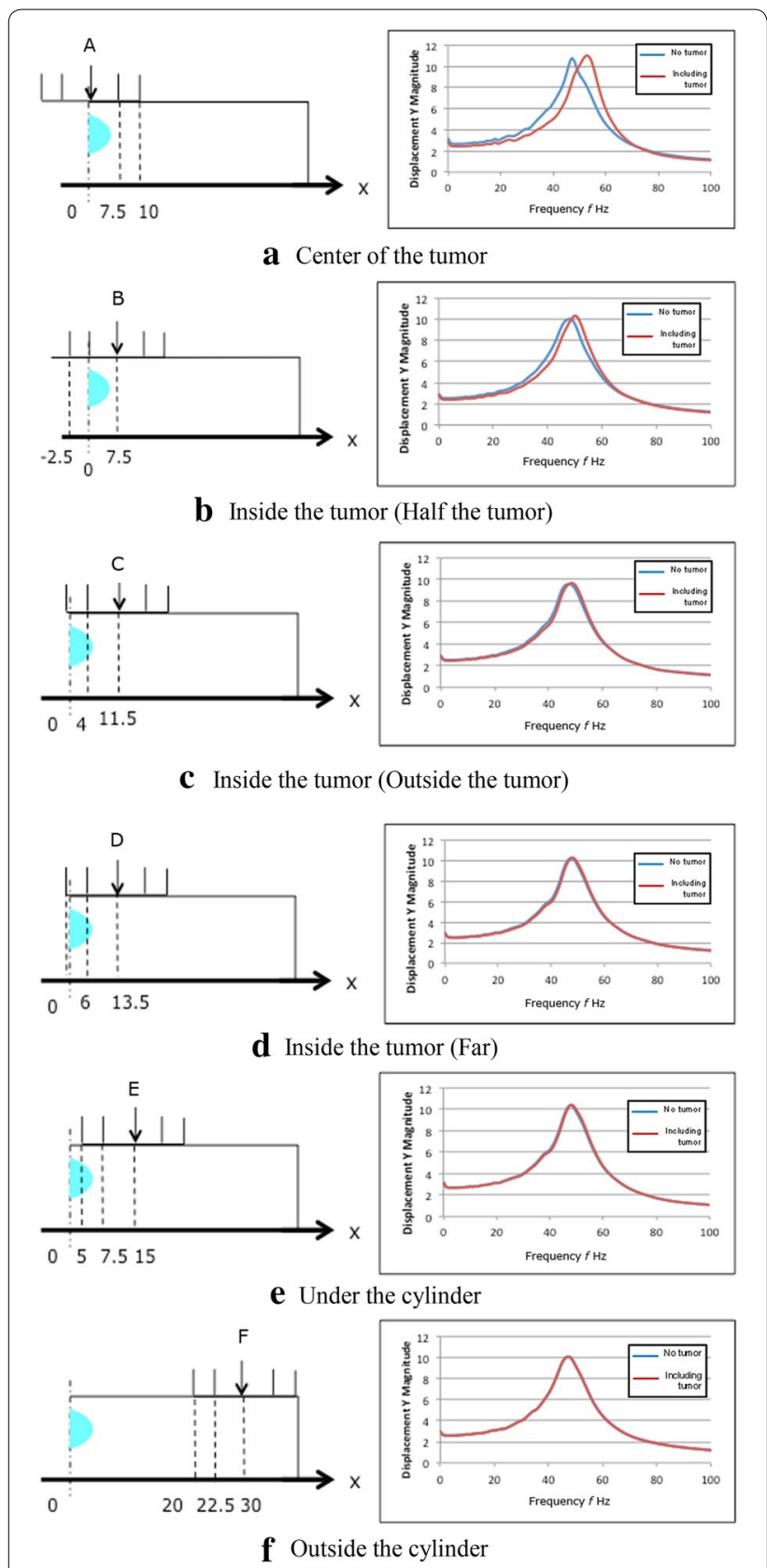

Fig. 13 Analysis results

difference in frequency would make difference between with and without cylinder. In this paper, we validated the feasibility of the pushing cylinder method in an experiment and performed analyses in a breast model.

The vibration analysis method shows the feasibility to distinguish between a normal tissue or tumor, however, we did not validate to distinguish between a benign or malignant tumor. At the next stage, it is necessary to validate the feasibility of distinction of the tumor types. Although there is meaning in revealing that the difference frequency was $5.5 \mathrm{~Hz}$ using vibration, compared with the previous work [18], $5.5 \mathrm{~Hz}$ is too small to detect the tumor type. We will develop the tumor type discrimination algorithm using known tumor size and depth information.

In terms of the tumor depth, it's necessary to carry out the FEM experiment under the condition that tumor depth is changing. It's expected that the frequency change would be similar to the experimental results of the paper because we revealed the frequency changes according to the change of the distance between vibration point and tumor.

Tendency of the result with and without cylinder is similar between phantom and FEM experiment. However, peak frequency is different because the load in FEM is different from the impact of the air-compression. We need more accurate impact model on FEM. Or we will propose another load method to model it easier instead of the air-compressor.

Furthermore, not only tumor types, but the tumor size should also be verified to change the frequency. In addition, the phantom shape is just cube. It's necessary to analyze the optimized shape suited for actual breast shape. Moreover, we should compare with the ultrasonic method and radiography such as mammography, CT scan and PET-CT. These imaging methods are easy to find the tumor presence and position. However, it is difficult to distinguish the tumor types. The vibration analysis method would be inferior to the imaging method in discovery of the tumor presence and position, but would be superior in distinction of the tumor types.

\section{Conclusions}

We proposed a novel diagnostic method to distinguish the tissue types such as normal tissue or tumor according to the difference in peak frequency during vibration. We pushed a cylinder and vibrated the skin from the center of the cylinder to reduce the distance to the tumor and render the tumor more difficult to move within the tissue. Our primary conclusions were:

(1) In our experiments, we vibrated the silicon with and without the simulated tumor, using air injection to investigate changes in measurement compliance with and without pushing the cylinder into a phantom breast model. Results showed that the displacement difference rate was more than $50 \%$ at a frequency of $130 \mathrm{~Hz}$ with the cylinder.

(2) Using finite element analysis, we constructed a simulated breast model based on a real phantom model. With the cylinder, there was a displacement difference rate of greater than $30 \%$ between tumor and no tumor. 
(3) From finite element analysis, displacement changed based on the distance between the tumor and load point. The closer the load point was to the tumor, the greater the displacement difference in the peak frequency between samples with and without a tumor. At the center of the tumor, differences in peak frequencies with and without the tumor were $5.5 \mathrm{~Hz}$.

Overall, we concluded that the vibration analysis with a pushing cylinder method is useful to distinguish the tissue types. This study is the first stage, but we will distinguish between a benign or malignant tumor in future work. Furthermore, we will compare with the imaging device such as MRI, ultrasonic, mammography, CT and PET-CT.

\section{Acknowledgements}

The authors would like to thank Waseda University in Japan by offering the funding support for this publication. Furthermore, we thank the Editage Group (https://www.editage.com/) for editing a draft of this manuscript.

\section{Authors' contributions}

SM conceived the study and drafted the manuscript. HI and YS carried out all experiments and analyzed the data. AT, VP and TM participated in the research design and sequence alignment. All authors read and approved the final manuscript.

\section{Funding}

This work was supported in part by JSPS KAKENHI Grant Number JP18K18405.

\section{Availability of data and materials}

The datasets supporting the conclusions of this article are included within the article.

\section{Competing interests}

The authors declare that they have no competing interests.

\section{Author details}

${ }^{1}$ Department of Modern Mechanical Engineering, Waseda University, 3-4-1 Okubo, Shinjuku, Tokyo 169-8555, Japan. ${ }^{2}$ Department of Aeronautics and Astronautics, Tokyo Metropolitan University, 6-6 Asahigaoka Hino, Tokyo 191-0065, Japan.

Received: 8 September 2018 Accepted: 20 August 2019

Published online: 28 August 2019

\section{References}

1. Hipwell JH, Vavourakis V, Han L, Mertzanidou T, Eiben B, Hawkes DJ (2016) A review of biomechanically informed breast image registration. Phys Med Biol 61(2):R1-R31

2. Samani A, Zubovits J, Plewes D (2007) Elastic moduli of normal and pathological human breast tissues: an inversion-technique-based investigation of 169 samples. Phys Med Biol 52(6):1565-1576

3. Silverstein M et al (1995) Prognostic classification of breast ductal carcinoma-in-situ. Lancet 345(8958):1154-1157

4. Siriaunkgul S, Tavassoli FA (1993) Invasive micropapillary carcinoma of the breast. Mod Pathol 6(6):660-662

5. Lazebnik M et al (2007) A large-scale study of the ultrawideband microwave dielectric properties of normal, benign and malignant breast tissues obtained from cancer surgeries. Phys Med Biol 52(20):6093-6115

6. Norris H, Taylor H (1965) Prognosis of mucinous (Gelatinous) carcinoma of the breast. Cancer 18(7):1056-1061
7. Stavros AT, Thickman D, Rapp CL, Dennis MA, Parker SH, Sisney GA (1995) Solid breast nodules: use of sonography to distinguish between benign and malignant lesions. Radiology 196(1):123-134

8. Elshemey WM, Elsharkawy WB (2009) Monte Carlo simulation of X-ray scattering for quantitative characterization of breast cancer. Phys Med Biol 54(12):3773-3784

9. Heyes GJ, Mill AJ, Charles MW (2009) Mammography-oncogenecity at low doses. J Radiol Prot 29(2A):A123-A132

10. Karimi H, Fenster A, Samani A (2013) A novel fast full inversion based breast ultrasound elastography technique. Phys Med Biol 58(7):2219-2233

11. Conley RH et al (2015) Realization of a biomechanical model-assisted image guidance system for breast cancer surgery using supine MRI. Int J Comput Assist Radiol Surg 10(12):1985-1996

12. Tsalach A, Steinberg I, Gannot I (2014) Tumor localization using magnetic nanoparticle-induced acoustic signals. IEEE Trans Biomed Eng 61(8):2313-2323

13. Palacio-Torralba J et al (2015) Quantitative diagnostics of soft tissue through viscoelastic characterization using time-based instrumented palpation. J Mech Behav Biomed Mater 41:149-160

14. Sun LZ, Wang ZG, Liu Y, Wang G (2009) Elastography method for reconstruction of nonlinear breast tissue properties. Int J Biomed Imaging 2009:6

15. Wang ZG, Liu Y, Wang G, Sun LZ (2011) Nonlinear elasto-mammography for characterization of breast tissue properties. Int J Biomed Imaging 2011:5

16. Ozcan MU, Ocal S, Basdogan C, Dogusoy G, Tokat Y (2011) Characterization of frequency-dependent material properties of human liver and its pathologies using an impact hammer. Med Image Anal 15(1):45-52

17. Kashif AS, Lotz TF, Heeren AMW, Chase JG (2013) Separate modal analysis for tumor detection with a digital image elasto tomography (DIET) breast cancer screening system. Med Phys 40(11):113503

18. Yamakawa M, Shiina T (2012) Tissue viscoelasticity imaging using vibration and ultrasound coupler gel. Jpn J Appl Phys 51(7S):07GF12

19. Russell N et al (2017) Retrospective methods to estimate radiation dose at the site of breast cancer development after Hodgkin Iymphoma radiotherapy. Clin Transl Radiat Oncol 7:20-27. https://doi. org/10.1016/j.ctro.2017.09.004

20. Misdorp W (2002) Tumors of the mammary gland. Tumors Domest Anim. https://doi.org/10.1002/9780470376928.ch12

21. Wiseman BS, Werb Z (2009) Stromal effects on mammary gland development and breast cancer. Science 296(5570):1046-1049

22. Kobayashi Y et al (2012) Enhanced targeting in breast tissue using a robotic tissue preloading-based needle insertion system. IEEE Trans Robot 28(3):710-722

23. Godavarty A et al (2004) Diagnostic imaging of breast cancer using fluorescence-enhanced optical tomography: phantom studies. J Biomed Opt 9(3):488

24. Mou X, Chen X (1998) Physics in medicine \& biology X-ray characterization of breast phantom materials. Phys Med Biol 43:1367-1377

25. Burfeindt MJ et al (2012) MRI-derived 3-D-printed breast phantom for microwave breast imaging validation. IEEE Antennas Wirel Propag Lett $11: 1610-1613$

26. Hebden JC et al (2001) Three-dimensional time-resolved optical tomography of a conical breast phantom. Appl Opt 40(19):3278

27. Wellman PS, Howe RD, Dalton E, Kern KA (1999) Breast tissue stiffness in compression is correlated to histological diagnosis. Tech Report Harvard BioRobotics Lab, pp: 1-15

28. Krouskop TA, Wheeler TM, Kallel F, Garra BS, Hall T (1998) Elastic moduli of breast and prostate tissues under compression. Ultrason Imaging 20(4):260-274

\section{Publisher's Note}

Springer Nature remains neutral with regard to jurisdictional claims in published maps and institutional affiliations. 